\title{
Bars formed in galaxy merging and their classification with deep learning
}

\author{
M. K. Cavanagh and K. Bekki
}

ICRAR M468, The University of Western Australia, 35 Stirling Hwy, Crawley, WA 6009, Australia
e-mail: 21727193@student. uwa . edu. au

Received 15 March 2020 / Accepted 24 June 2020

ABSTRACT

\begin{abstract}
Context. Stellar bars are a common morphological feature of spiral galaxies. While it is known that they can form in isolation, or be induced tidally, few studies have explored the production of stellar bars in galaxy merging. We look to investigate bar formation in galaxy merging using methods from deep learning to analyse our $N$-body simulations.

Aims. The primary aim is to determine the constraints on the mass ratio and orientations of merging galaxies that are most conducive to bar formation. We further aim to explore whether it is possible to classify simulated barred spiral galaxies based on the mechanism of their formation. We test the feasibility of this new classification schema with simulated galaxies.

Methods. Using a set of 29400 images obtained from our simulations, we first trained a convolutional neural network to distinguish between barred and non-barred galaxies. We then tested the network on simulations with different mass ratios and spin angles. We adapted the core neural network architecture for use with our additional aims.

Results. We find that a strong inverse relationship exists between the mass ratio and the number of bars produced. We also identify two distinct phases in the bar formation process; (1) the initial, tidally induced formation pre-merger and (2) the destruction and/or regeneration of the bar during and after the merger.

Conclusions. Mergers with low mass ratios and closely-aligned orientations are considerably more conducive to bar formation compared to equal-mass mergers. We demonstrate the flexibility of our deep learning approach by showing it is feasible to classify bars based on their formation mechanism.
\end{abstract}

Key words. galaxies: general - galaxies: formation - galaxies: evolution

\section{Introduction}

Central bar structures are a common feature of most spiral galaxies. Around $30 \%$ of all spiral galaxies are understood to be strongly barred (Sellwood \& Wilkinson 1993), while overall more than $50 \%$ of spiral galaxies typically contain features indicative of a central bar structure (Eskridge \& Frogel 1999). Bars are found to be even more prevalent when observed in the infrared (Eskridge et al. 2000). Previous studies regarding the significance and physical properties of central bar structures have found that bars play an important role in both the long- and short-term evolution of spiral galaxies. It is known that stellar bars have a profound impact on the dynamics and evolution of spiral galaxies (Little \& Carlberg 1991; Sellwood \& Wilkinson 1993; Athanassoula 2005; Vera et al. 2016), as well as the subsequent evolution of the interstellar medium (Mayer et al. 2006; Fanali et al. 2015). Bars also drive interactions between the stellar disc and dark matter halo (Athanassoula 2003; Dalcanton et al. 2004; Sellwood 2014), redistribute angular momentum within the disc (Athanassoula 2005; Aguerri et al. 2009), and accelerate the formation of a spiral structure (LyndenBell \& Kalnajs 1972; Pfenniger \& Friedli 1991), central bulges (Kormendy \& Kennicutt 2004; Gadotti 2011), and other morphological features (Rautiainen et al. 2002; Regan \& Teuben 2003).

In this work we consider three models of bar formation; the self-gravitating isolated model (also known as the spontaneous model), the tidal interaction model, and the galaxy merger model using $N$-body simulations based on the previous work of Bekki (2013), Bekki et al. (2019). The physical conditions behind the formation of isolated bars and tidally-induced bars have already been well investigated (Hohl 1971; Little \& Carlberg 1991; Noguchi 1987; Barnes \& Hernquist 1992; Miwa \& Noguchi 1998; Berentzen et al. 2004); however, there has been limited to no research on the formation of bars in galaxy merging. Galaxy merging is an important factor in the evolution of galaxies (Conselice 2014), particularly as it affects star formation (Pearson et al. 2019) and the growth of galaxies (Cattaneo et al. 2011).

The primary purpose of this paper is to investigate the physical parameters behind the formation of stellar bars in galaxy merging. Studies such as Peirani et al. (2009) and Moetazedian et al. (2017) have shown that merging galaxies can induce bars prior to the collision, but we look to investigate the circumstances under which bars can emerge from the aftermath of a merger unscathed. We investigate the effect of the mass-ratio $m_{2}$ and spin angles $\theta_{1}, \theta_{2}$ of two merging galaxies on the incidence of bar formation (these are formally defined in Sect. 3.3). Aided by deep learning, we attempt to determine the physical parameters most conducive to bar formation. We propose a method to use convolutional neural networks (CNNs) to automatically classify the images from the results of our $N$-body galaxy simulations as either barred or non-barred. Such a method allows us to rapidly classify data and for data, which were otherwise considered intractable, to be analysed in a more practical time frame. Apart from the initial work needed to prepare and label the training data, the neural network is able to quickly test our simulation outputs.

In addition to our primary aim, we also look to investigate the ability of a CNN to discriminate between multiple mechanisms of bar formation: in other words, given an image of a galaxy, 
we strive to determine whether it was formed (a) in isolation, (b) due to the tidal interaction, or (c) during a galaxy merger. Since CNNs work by identifying features in data, this feasibility study is intended to determine whether a barred galaxy's current morphology is indicative of the means by which it was formed. If so, this has the potential to further accelerate large-scale surveys into the prevalence and nature of bars in the Universe.

The structure of the paper is as follows. We provide a brief background behind the three formation mechanisms and our neural network implementation in Sect. 2. We explain our simulation models and orbital parameters, as well as describe the process of our CNN-based analysis in Sect. 3. We present the results of our analysis in Sect. 4. In Sect. 5 we discuss our results, focusing on the effect of the mass ratio and spin angles of the overall bar formation process. In Sect. 6, we discuss our secondary aim of using a CNN to distinguish between multiple bar formation mechanisms, along with an evaluation of our CNN approach. Lastly, we summarise our key conclusions in Sect. 7.

\section{Bar formation mechanisms and deep learning}

\subsection{Bar formation mechanisms}

In this work we consider three mechanisms of bar formation: the isolated or self-gravitating model, the tidal interaction model and the galaxy merger model.

\subsubsection{Isolated model}

It is well known that large-scale self-gravitating discs are prone to instability unless certain conditions for stability are met (Toomre 1964). The first two-dimensional numerical simulation to investigate disc instabilities was conducted by Hohl (1971), who found that slow-growing large-scale non-axisymmetric disturbances eventually led to the formation of a central bar structure. Later simulations conducted in three dimensions were able to observe bars forming from initially axisymmetric discs, suggesting that the global instability is an inherent property of the disc regardless of whether or not the initial particle distributions are axisymmetric. The 3D simulation by Pfenniger (1984) found significant vertical instability despite the flat disc. Interestingly, this vertical instability can lead to dynamical buckling and the formation of boxy peanut-shaped bars (Raha et al. 1991). Sparke \& Sellwood (1987) note the difficulty for a completely isolated bar to survive indefinitely. Indeed, simulations show that such isolated bars can be destroyed due to large-scale accretion (Friedli \& Benz 1993). It has long been established that bars can form due to orbital resonances associated with global instabilities (Athanassoula et al. 1983; Contopoulos \& Grosbøl 1989; Pfenniger \& Friedli 1991). Several mechanisms have been proposed to explain the how these instabilities result in bar formation, such as the swing amplification of propagating density waves (Goldreich \& Lynden-Bell 1965; Julian \& Toomre 1966) to the Lynden-Bell mechanism of resonant orbits (Lynden-Bell 1979).

\subsubsection{Tidal model}

The isolated model is a case of internally-induced bar formation. However, bars are more frequently observed in dense environments such as clusters (Elmegreen et al. 1990) with characteristics that differ from isolated bars, such as a slower pattern speed and differences in the location of inner Lindblad resonances (Miwa \& Noguchi 1998). It has long been recognised that tidal interactions between galaxies can influence morphology and bar formation (Toomre \& Toomre 1972; Athanassoula 1999; Barnes \& Hernquist 1992). The pioneering simulation of (Noguchi 1987) investigating the effects of tidal deformation of galaxy discs and showed that non-axisymmetric instabilities can be induced throughout the disc, leading to the formation of a stellar bar. Importantly, the strength of this bar was found to depend on many parameters, such as the mass ratio between the perturbed and peturbing galaxies, as well as bulge and halo ratios (Noguchi 1987). Tidal interactions are sufficient to support the evolution of the bar, with the capability to regenerate the bar should it weaken due to its inherent instability (Berentzen et al. 2004). This can be simulated in the case of low-mass satellite galaxies interacting with their host, where the properties of the host's tidal bar are found to be independent of the number of tidal interactions (Moetazedian et al. 2017).

\subsubsection{Merger model}

Galaxy merging is a form of direct galaxy-galaxy interaction where two galaxies come into contact. However, the large timescale over which such mergers take place necessitate the use of numerical simulations in order to investigate morphological changes and the possibility of bar formation. Previous research has shown that significant changes to morphology and star formation can be triggered due to galaxy merging (Hernquist \& Mihos 1995). Galaxy merging is a particularly important driver of star formation (Lin et al. 2007; Bridge et al. 2007; Kaviraj et al. 2009, 2015), and also plays a key role in the morphological evolution of galaxies (Conselice 2014). The traditional view is that many galaxy mergers, particularly major mergers where the masses of the colliding galaxies are approximately equal, destroy the overall galaxy discs with the remnants eventually combining to form early-type elliptical galaxies (Toomre \& Toomre 1972; Negroponte \& White 1983; Barnes 1996). Despite this, more recent simulations have shown that spiral galaxies can emerge from galaxy mergers (Springel \& Hernquist 2005) as well as the formation of stellar bars (Di Matteo et al. 2010). Our work aims to guide future exploration of the latter, for although numerical simulations have demonstrated that bars can be formed in galaxy merging (Peirani et al. 2009; Lotz et al. 2010), the processes behind bar formation in mergers are not well understood. It is important to stress that Peirani et al. (2009) used a merger to model a particular galaxy's stellar bar, while in this work we focus on the mechanism of bar formation through simulating many hundreds of mergers in order to systematically test the effects of changing the mass ratio $m_{2}$ and spin angles $\theta_{1}, \theta_{2}$.

\subsection{Convolutional neural networks}

In the literature, a variety of machine learning methods have been applied in the field of astronomy, ranging from artificial neural networks with the aim to classify galaxy morphology (Naim et al. 1995; Calleja \& Fuentes 2004; Dieleman et al. 2015; Consolandi 2016; Abraham et al. 2018; Diaz et al. 2019), to analysing largescale simulations (Huertas-Company et al. 2019) and to generalpurpose training programmes (Vasconcellos et al. 2011; Graff et al. 2014). These applications of deep learning for morphological classification are not restricted to the optical, with Wu et al. (2018a) and Lukic et al. (2019) investigating morphological classification of radio sources.

In this work, we utilise convolutional neural networks to determine the presence of a bar in an image of a galaxy, and 
later classify a bar according to the mechanism with which it was formed. A CNN (LeCun et al. 1998) is a type of neural network that is especially useful for image analysis due to its use of feature maps (LeCun et al. 2015). These feature maps excel at finding high-level features of an image, which are key to the ability to discriminate one image from another (Haykin 2009).

A general schematic showing how a CNN processes an input image is shown in Fig. 1. There exist many different CNN architectures in the literature (LeCun et al. 2015) that differ in the structure, type and quantity of component layers, as well as utilising different weighting and activation functions. However, memory and (computational) time limitations ultimately result in some degree of compromise between complexity and overall performance.

Our CNN architecture is based on Bekki et al. (2019), with the goal of inputting a $50 \times 50$ density map and outputting a label to indicate whether or not a bar was detected. We used Keras for the CNN construction (Chollet 2015). There are 2 convolution layers (Conv2D), a max-pooling layer (MaxPooling2D), two dropout layers (Dropout), a flatten layer (Flatten) and finally two dense layers (Dense) that are fully-connected (including the output layer). The role of the convolution layers is to extract the high- level features of the preceeding layer. This is done via the use of a kernel filter that performs a matrix operation, progressively scanning through the input layer. In our network, each Conv2D layer uses a $3 \times 3$ kernel. Our $50 \times 50$ input image is mapped into 32 separate convolved feature maps via the first Conv2D layer. These maps are then further convolved into 64 separate feature maps via the second Conv2D layer. Each of these convolutional layers can be thought of as a layer of abstraction, enabling higher-level features to be extracted.

The next major part of the architecture is the MaxPooling2D layer. In general, a pooling layer is designed to average out the convolved feature maps through downsampling. Instead of averaging, MaxPooling2D returns local maxima. This achieves two major goals: firstly, it decreases the computational complexity, while secondly the max-pooling retains the dominant features from the convolved feature map. The Flatten layer is used to vectorise the feature maps, while the Dropout layers are used to avoid over-fitting by ignoring a fraction of the preceding nodes. The final fully-connected Dense layer (before the output layer) thus combines the flattened nodes. From here on, the architecture is similar to that of a basic neural network; the weights and biases of the Dense layer nodes can be tweaked in order to associate different high-level features with the desired output category. For our CNN, we used the ReLu activation function for the convolution and dense layers, whilst the final output layer used a softmax (normalised exponential) activation with the categorical crossentropy loss function. For training, we used the ADADELTA adaptive learning rate method, developed by Zeiler (2012). This core architecture was used for both parts of our investigation, the only crucial difference being that the CNN used to test multiple bar formation mechanisms had an extended output layer in order to account for the new labelling scheme.

\subsection{Differences with existing bar detection techniques}

There are several existing methods in which to both detect bars and analyse their physical characteristics; ellipse fitting of the galaxy isophotes, Fourier analysis of the (azimuthal) luminosity profile and a decomposition of the surface brightness distribution (Prieto et al. 2001). These methods involve significant pre and post-processing, and often require access to additional data such as spectroscopic flux measurements and velocity dispersion maps.
Hence these methods cannot readily determine the presence of a bar from mere images alone. Another method involves directly analysing the image of a galaxy using Fourier techniques. These methods are well-known to detect the presence of stellar bars and spiral arms, as well as characterise their shape and strength (Garcia-Gómez \& Athanassoula 1991; Aguerri et al. 1998; Barberà et al. 2004; Garcia-Gómez et al. 2017).

CNNs are similar to Fourier transforms in this regard, as their core operation revolves around the detection of high-level features. Morphological classification is thus a natural application. Another difference with our CNN approach is that it avoids much of the extra image processing needed in traditional methods more suited to observational data (see Prieto et al. 2001). Furthermore, the CNN can be trained on images with high inclination, whereas methods similar to Garcia-Gómez et al. (2017) must perform a de-projection.

The bottleneck for CNNs lies with the training. Once trained, CNNs can quickly analyse images and output a label corresponding to what it has detected the image to be. Existing methods are tailored for real-world data, whereas our CNN is specifically designed to work with the output of our galaxy simulations. It is computationally expensive to accurately model luminosity profiles. Our CNN works with the output of dynamical $N$-body simulations, allowing it to be trained and tested on a larger dataset than we would otherwise have been able to compile. Our CNNbased approach to bar detection is merely tackling the problem from a different angle (i.e. feature detection with CNNs) where all we are given is the image of the galaxy. It is not designed to supplant nor contest any existing method; rather it is a tool well suited to our aim of analysing many galaxy simulations in order to constrain parameters based on how many bars are detected.

\section{Simulation and implementation}

\subsection{Structure and kinematics of the stellar disc}

We consider merging of two spiral galaxies with various bulgeto- disc-ratios and baryonic mass fractions in order to investigate how stellar bars can be induced during galaxy merging. The total masses of dark matter halo, stellar disc, gas disc, and bulge of a disc galaxy are denoted as $M_{\mathrm{h}}, M_{\mathrm{s}}, M_{\mathrm{g}}$, and $M_{\mathrm{b}}$, respectively. In these preliminary works, we only show the results for models with no gas $\left(f_{\mathrm{g}}=M_{\mathrm{g}} / M_{\mathrm{s}}=0\right)$ The bulge-to-disc-ratio is defined as $M_{\mathrm{b}} / M_{\mathrm{s}}$ and represented by the parameter $f_{\mathrm{b}}$. The key parameters in the present study are $M_{\mathrm{h}}, f_{\text {bary }}$, and $f_{\mathrm{b}}$. We adopt the density distribution of the NFW halo Navarro et al. (1996), as suggested from CDM simulations, to describe the initial density profile of the dark matter halo in a disc galaxy:

$\rho(r)=\frac{\rho_{0}}{\left(r / r_{\mathrm{s}}\right)\left(1+r / r_{\mathrm{s}}\right)^{2}}$,

where $r, \rho_{0}$, and $r_{\mathrm{s}}$ are the spherical radius, the characteristic density of the halo, and the scale length of the halo respectively. The $c$-parameter ( $c=r_{\text {vir }} / r_{\mathrm{s}}$, where $r_{\text {vir }}$ is the virial radius of a dark matter halo) along with $r_{\text {vir }}$ are chosen appropriately for a given dark halo mass $\left(M_{\mathrm{dm}}\right)$ by using the $c-M_{\mathrm{h}}$ relation for $z=0$ predicted by recent cosmological simulations, for example Neto et al. (2007).

The bulge of the disc galaxy has a size $R_{\mathrm{b}}$ and a scalelength $R_{0, \mathrm{~b}}$, and is represented by the Hernquist density profile. The bulge is assumed to have an isotropic velocity dispersion, with radial velocity dispersion given by the Jeans equation for a spherical system (Binney \& Tremaine 2008). The bulge-to-disc 


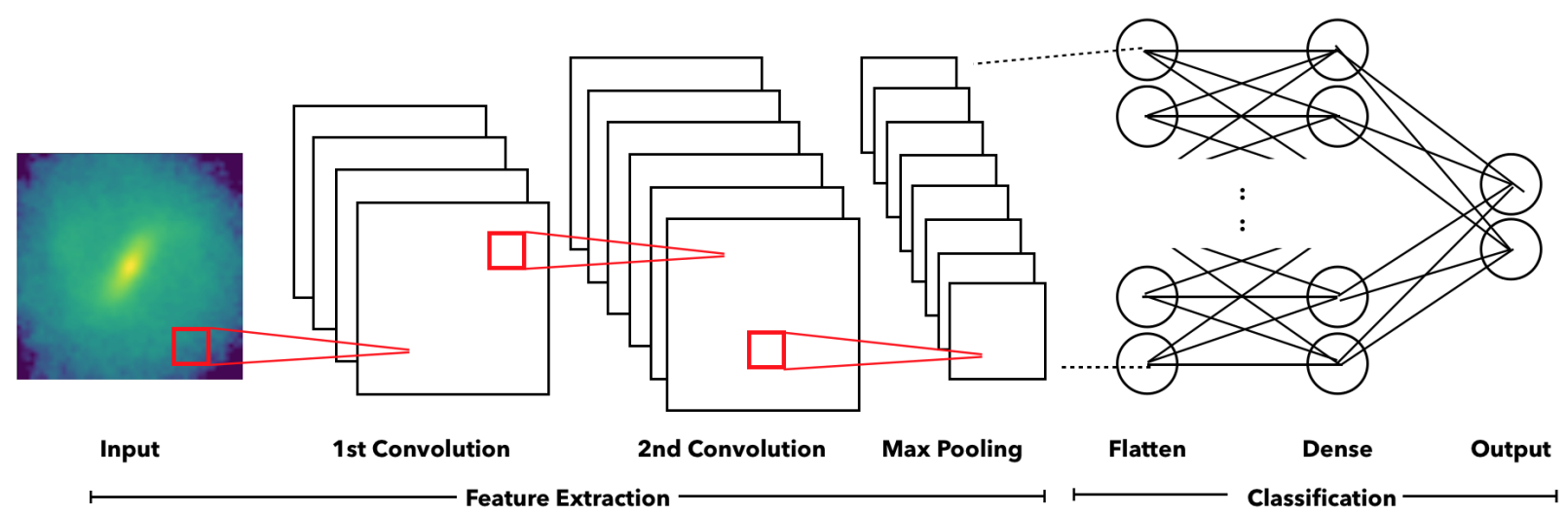

Fig. 1. Schematic overview of the general architecture of a convolutional neural network. The input image is mapped into various feature maps via convolutions, followed by a pooling layer that performs local averaging and sub-sampling. The final layer is a list of outputs that, in our case, correspond to whether a bar is present or not.

ratio $\left(f_{\mathrm{b}}=M_{\mathrm{b}} / M_{\mathrm{d}}\right)$ of the disc galaxy is a free parameter ranging from 0 (pure disc galaxy) to 1 . Our "MW-type" models are those with $f_{\mathrm{b}}=0.17$ and $R_{\mathrm{b}}=0.2 R_{\mathrm{s}}$, where $R_{\mathrm{s}}$ is the stellar disc size of a galaxy. We adopt the mass-size scaling relation of $R_{\mathrm{b}}=C_{\mathrm{b}} M_{\mathrm{b}}^{0.5}$ for bulges such that we can determine $R_{\mathrm{b}}$ for a given $M_{\mathrm{b}}$. The value of $C_{\mathrm{b}}$ is determined in order for $R_{\mathrm{b}}$ to be $3.5 \mathrm{kpc}$ for $M_{\mathrm{b}}=10^{10} M_{\odot}$ (this corresponds to the mass and size of the MW's bulge).

The radial $(R)$ and vertical $(Z)$ density profiles of the stellar disc are assumed to be proportional to $\exp \left(-R / R_{0}\right)$ with scale length $R_{0}=0.2 R_{\mathrm{S}}$ and to $\operatorname{sech}^{2}\left(Z / Z_{0}\right)$ with scale length $Z_{0}=0.04 R_{\mathrm{s}}$, respectively. In the present model for the MW-type, the exponential disc has $R_{\mathrm{s}}=17.5 \mathrm{kpc}$. In addition to the rotational velocity caused by the gravitational field of disc, bulge, and dark halo components, the initial radial and azimuthal velocity dispersions are assigned to the disc component according to the epicyclic theory (Toomre 1964) with Toomre's parameter set to $Q=1.5$. The vertical velocity dispersion at a given radius is set to be 0.5 times as large as the radial velocity dispersion at that point.

The total numbers of particles in a fiducial model with $f_{\mathrm{b}}=$ 0.167 is 216700 , though for other models it depends on $f_{\mathrm{b}}$. The mass resolution for each of the models in the present study is $1.2 \times 10^{6} M_{\odot}$. The gravitational softening length for each component is determined by the number of particles used for each component, as well as by the size of the distribution (e.g. $R_{\mathrm{S}}$ and $\left.r_{\text {vir }}\right)$. It is set to be $320 \mathrm{pc}$, which is much finer than $1-2 \mathrm{kpc}$ spatial resolutions used for the image analysis in the present study. These spatial and mass resolutions are not particularly high; this is predominantly because we have to run a large number of models within a limited amount of GPU computing time allocated for this project. We believe that the adopted resolutions and subsequent galaxy images are sufficient for use with the CNNs. A summary of our simulation parameters and features is provided in Table 1 .

\subsection{Orbital configurations for galaxy merging}

In all of the merger simulations with different mass-ratios of two discs $\left(m_{2}\right)$, the orbit of the two discs is set to be initially in the $x y$ plane. The initial distance between the centre of mass of the two discs, the pericentre distance, and the circular velocity factor $\left(f_{\mathrm{v}}\right)$ for the companion galaxy are set to be $8 R_{\mathrm{s}}, 2 R_{\mathrm{s}}$, and 0.5 respectively for all models. For this study, we have set the velocity of the two disc such that all orbits are prograde
Table 1. Description of the basic parameter values for the MW-type disc model.

\begin{tabular}{lc}
\hline \hline Physical properties & Parameter values \\
\hline Total halo mass (galaxy) & $M_{\mathrm{dm}}=1.0 \times 10^{12} M_{\odot}$ \\
DM structure (galaxy) & $\mathrm{NFW}$ profile \\
Galaxy virial radius (galaxy) & $R_{\mathrm{vir}}=245 \mathrm{kpc}$ \\
$c$ parameter of galaxy halo & $c=10$ \\
Stellar disc mass & $M_{\mathrm{s}}=6.0 \times 10^{10} M_{\odot}$ \\
Stellar disc size & $R_{\mathrm{s}}=17.5 \mathrm{mkpc}$ \\
disc scale length & $R_{0}=3.5 \mathrm{kpc}$ \\
Gas fraction in a disc & $f_{\mathrm{g}}=0$ \\
Bulge mass & $M_{\mathrm{b}}=10^{10} M_{\odot}$ \\
Bulge size & $R_{\mathrm{b}}=3.5 \mathrm{kpc}$ \\
Mass resolution & $1.2 \times 10^{6} M_{\odot}$ \\
Size resolution & $320 \mathrm{pc}$ \\
Spatial resolution for image analysis & $1-2 \mathrm{kpc}$ \\
Star formation & Not included \\
Chemical evolution & Not included \\
Dust evolution & Not included \\
\hline
\end{tabular}

and initially confined to the $x y$ plane. We define $f_{\mathrm{v}}$ as the ratio of the total 3D velocity of the companion galaxy (with respect to the primary galaxy) to the circular velocity at the distance of the companion from the centre of the primary galaxy. The azimuthal angle $\phi$ is measured from the $x$-axis to the projection of the angular momentum vector of the disc onto the $x-y$ plane. This is set to be 0 for both disc galaxies, as it is not an important parameter in the present study. The time when the progenitor discs merge completely and reach the dynamical equilibrium is typically less than 12.0 in our units for most of models.

\subsection{Definition of mass ratio and spin angles}

We define the mass ratio $m_{2}$ as the ratio of the mass of the companion galaxy to that of the parent. We refer to a minor merger as a merger with $m_{2} \approx 0.1$, and a major merger as that with $m_{2} \approx 1$. We also refer to intermediate mass mergers as those with $m_{2}$ between 0.1 and 1 . The spin of each galaxy is specified by two angles, $\theta_{i}$, where the subscript $\mathrm{i}$ is used to identify each galaxy ( $\theta_{1}$ for the primary galaxy, $\theta_{2}$ for the companion). The spin angles $\theta_{i}$ are defined as the angle between the $z$-axis and 


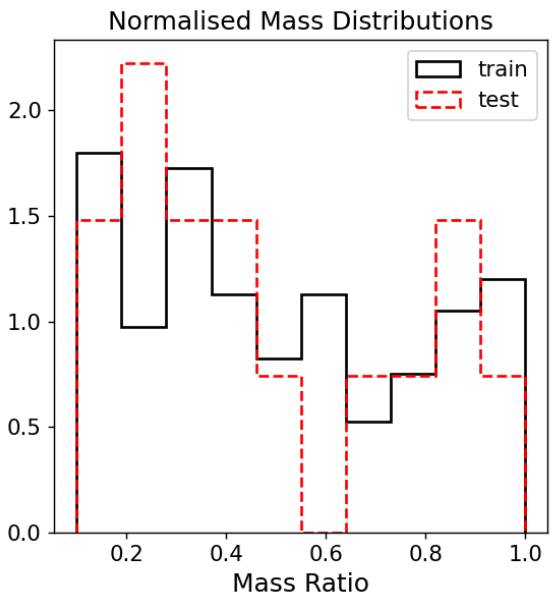

Fig. 2. Normalised histograms showing the distributions of mass ratios (left) and $\theta_{1}-\theta_{2}$ (right) for the training and testing sets. the vector of the angular momentum of the disc. In our present study, we vary these angles between -180 and 180 degrees.

\subsection{Simulation output and training data}

The raw density output from our simulations comprised a list of local density values ordered by pixel for each of the 200 different viewing angle orientations, with separate files for each model and time-step. We derive 2D density maps of our simulated galaxies for $R \leq R_{\mathrm{s}}$. We first divide the stellar disc $\left(R \leq R_{\mathrm{s}}\right)$ of the galaxy into a $50 \times 50$ grid mesh and estimate the mean stellar mass density at each mesh point as follows:

$\Sigma_{i, j, 0}=\frac{1}{\Delta R_{i, j}{ }^{2}} \sum_{k=1}^{N_{i, j}} m_{k}$,

where $\Delta R_{i, j}, N_{i, j}$, and $m_{k}$ are the mesh size at the mesh point $(i, j)$, the total number of stellar particles in the mesh, and the mass of a stellar particle, respectively. These values were normalised to obtain the normalised density maps. The mesh size is set to $0.04 R_{\mathrm{s}}$, which roughly corresponds to $0.7 \mathrm{kpc}$ for an MW-type disc galaxy.

To create the training data for our $\mathrm{CNN}$, the raw density data was stitched together to create $50 \times 50$ images (corresponding to the normalised density maps). Thus the input layer to our neural network consisted of a linear array of 2500 pixel values corresponding to the $50 \times 50$ density maps. This is how the individual images were tested with the neural network. For each individual galaxy model, we obtained a total of 200 images that differed in orientation and inclination. This is to ensure that the training set includes images of bars with different projections and orientations, and to mirror real life data.

These density maps, totalling 29400 total images, were combined to create the training set for the CNN. Each of these training images were manually assigned a label (corresponding to a bar or no-bar) through visual inspection in order to create the input-output pairs with which to train the CNN. The visual classification was conducted by both authors, restricted to only the face-on images for each model. On average, 1 in 20 images were classified differently by each observer. These were overwhelmingly galaxies with oblate bulges, where the distinction between a bar and a bulge is not so objectively clear. As a result, we estimate that the overall labelling of the training set is $95 \%$ accurate (i.e. there is an overall uncertainty of around 5\%). A key aspect of any training set is that there should be a roughly equal number of samples for each of the classification categories, otherwise there is a bias towards the category with the greater number of samples. In our case, that means ensuring the number of barred and non-barred galaxy samples are about the same. Many of our simulations were thus manually checked to see whether or not a bar had been formed. The training set was split into separate training and validation sets such that $80 \%$ of the samples were used to train, with the remaining $20 \%$ used for validation. The validation data was randomly partitioned from the main sample set. Figure 2 shows the distribution of mass ratio and angular separation $\left(\theta_{1}-\theta_{2}\right)$ for the training and test sets. Ideally these should be uniform, although there is a slight bias towards samples with lower mass ratios samples.

We define the prevalence of bar formation in a given model as the fraction of the 200 images that returned a label of "bar" when tested with the CNN. We performed these tests for density outputs with different values of mass-ratio $m_{2}$ and spin- angles $\theta_{1}$ and $\theta_{2}$, for different galaxy models with different bulge-to-disc ratios and dark matter contents. A full description of the exact parameters used for each model is provided in Table 2.

\subsection{Training the network}

The CNN was coded using Keras (Chollet 2015), a high-level deep learning application programming interface (API), running on TensorFlow, a machine learning framework. Training was conducted on the ICRAR's Pleiades cluster, making use of a Nvidia GTX1080. Here all 29400 images were used to train the $\mathrm{CNN}$, with training conducted in stages up to a combined total 1000 epochs. The training and validation accuracies, along with a confusion matrix, are presented in Fig. 3 .

The validation accuracy converged to between $98 \%$ and $99 \%$ within around 200 epochs, after which there was no further increase. Given that this is well above what would be expected due to the $5 \%$ uncertainty inherent in the training set, it is likely that there is some degree of overfitting. The batch size was set to 200 images, given that this corresponds to a single galaxy model. Modifying the batch size did not appreciably impact the final accuracy. Training for our secondary, multiple-bar classification $\mathrm{CNN}$ was also conducted on Pleiades, however this used a much smaller subset of 4800 images.

To achieve our primary goal of constraining bar formation in galaxy merging, we conducted simulations with varying massratios and spin angles $\theta_{1}, \theta_{2}$ for both disc-dominated and bulgedominated initial discs. The output from these simulations were then tested with the CNN. Since each simulation output has 200 associated density maps (each oriented differently with respect 

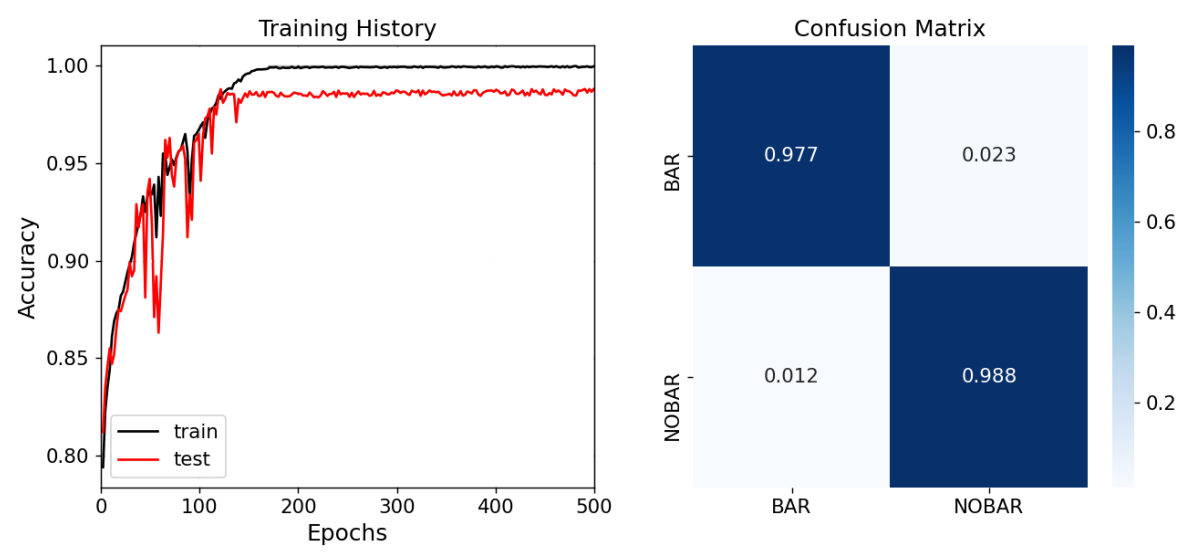

Fig. 3. Training and testing (validation) accuracies against training time (left), as well as the confusion matrix (right) for the main $\mathrm{CNN}$.

Table 2. Dark matter, stellar, bulge components, and bulge-to-disc ratios for each of our six models.

\begin{tabular}{lcccc}
\hline \hline Model & $\begin{array}{c}\text { Dark matter } \\
10^{12} M_{\odot}\end{array}$ & $\begin{array}{c}\text { Stellar mass } \\
10^{10} M_{\odot}\end{array}$ & $\begin{array}{c}\text { Bulge mass } \\
10^{10} M_{\odot}\end{array}$ & $\begin{array}{c}\text { Bulge-to-disc } \\
\text { Ratio }\end{array}$ \\
\hline m1 & 1 & 6 & 1 & 0.167 \\
m2 & 1 & 3 & 0.5 & 0.167 \\
m3 & 1 & 1.8 & 0.3 & 0.167 \\
m4 & 1 & 6 & 3 & 0.5 \\
m5 & 1 & 6 & 6 & 1 \\
m6 & 1 & 6 & 0 & 0 \\
\hline
\end{tabular}

to the viewing axis), we define the prevalence of bar formation as the fraction of these 200 images that are classified as a bar by the CNN. That is, we define the bar fraction as:

$f_{\text {bar }}=N_{\text {bar }} / N$

where $N_{\text {bar }}$ are the number of images detected as showing a bar, and $N$ is the total number of images. We also refer to this as the bar probability, with the terms used interchangeably depending on the context. We ran several disc-dominated and bulge- dominated models, and tested the outputted data with our fully- trained CNN to determine the prevalence of bar formation as functions of mass ratio $m_{2}$ and spin angles $\theta_{1}, \theta_{2}$. Our secondary goal of using a CNN to distinguish between different bar formation mechanisms used a new CNN with the same core architecture with two key differences: it was trained on a different set of simulated data, and utilised a different labelling schema (4 categories instead of 2).

\section{Results}

\subsection{Mass ratio}

To investigate the effects of mass ratio, we ran several hundred simulations for each of the six galaxy models in Table 2 for a variable mass ratio ( $m_{2}$ between 0.1 and 1.0) as well as for the fixed mass ratios $m_{2}=0.1$ (corresponding to minor merging) and $m_{2}=1.0$ (corresponding to major merging). Each of the simulations were conducted with random spin angles such that $0 \leq \theta_{i} \leq 180$ degrees for $i=1,2$. These models are completely separate from those used to train the CNN. Instead, these models were classified by the fully-trained CNN.

Table 3 shows the detected bar fractions (i.e. as defined in Eq. (3)) for each of these three mass cases for our six galaxy simulation models (refer to Table 2 for their physical parameters). On average, the $\mathrm{CNN}$ detected more bars present in the
Table 3. Total bar fractions for the variable mass ratio $m_{2}=0.1$ to 1.0 and fixed mass ratios $m_{2}=0.1$ and $m_{2}=1.0$, for each of our six galaxy models.

\begin{tabular}{lccc}
\hline \hline Model & $\begin{array}{c}\text { Bar fraction } \\
m_{2}=0.1 \text { to } 1.0\end{array}$ & $\begin{array}{c}\text { Bar fraction } \\
m_{2}=0.1\end{array}$ & $\begin{array}{c}\text { Bar fraction } \\
m_{2}=1.0\end{array}$ \\
\hline $\mathrm{m} 1$ & $0.075 \pm 0.0037$ & $0.082 \pm 0.0041$ & $0.013 \pm 0.00065$ \\
$\mathrm{~m} 2$ & $0.064 \pm 0.0032$ & $0.073 \pm 0.0036$ & $0.029 \pm 0.0015$ \\
$\mathrm{~m} 3$ & $0.048 \pm 0.0024$ & $0.051 \pm 0.0026$ & $0.03 \pm 0.0015$ \\
$\mathrm{~m} 4$ & $0.12 \pm 0.006$ & $0.11 \pm 0.0055$ & $0.038 \pm 0.0019$ \\
$\mathrm{~m} 5$ & $0.089 \pm 0.0044$ & $0.091 \pm 0.0045$ & $0.057 \pm 0.00285$ \\
$\mathrm{~m} 6$ & $0.037 \pm 0.0019$ & $0.037 \pm 0.0019$ & $0.036 \pm 0.0018$ \\
\hline
\end{tabular}

aftermath of minor merging ( $m_{2}$ of the order of 0.1 ) as opposed to major merging $\left(m_{2} \sim 1.0\right)$. Figure 4 gives a visualisation of the bar probability as functions of the parameters $m_{2}, \theta_{1}, \theta_{2}, \theta_{1}-\theta_{2}$ and finally as a normalised probability density map corresponding to $\theta_{1}$ vs. $\theta_{2}$. Again we see a clear inverse relationship with mass ratio; lower mass ratios are more conducive to bar formation.

Figure 5 gives a more compact visualisation of the overall CNN classifications, showing the normalised histograms of all samples classified as bar or no-bar with respect to mass ratio and spin angles. As seen in Fig. 4, of those samples classified as bars, they generally have lower mass ratios and lower angular separation. Conversely, samples classified as no-bar tend to have mass ratios between 0.3 and 1.0, and are uniform with regards to angular separation. This latter result suggests that mass ratio is a more influential factor in the bar formation process.

It is important to stress that the results in Figs. 4 were obtained by running limited sets of random parameter combinations. Due to time limitations, it was impossible to cover all combinations of $m_{2}, \theta_{1}$ and $\theta_{2}$, but there are enough data 

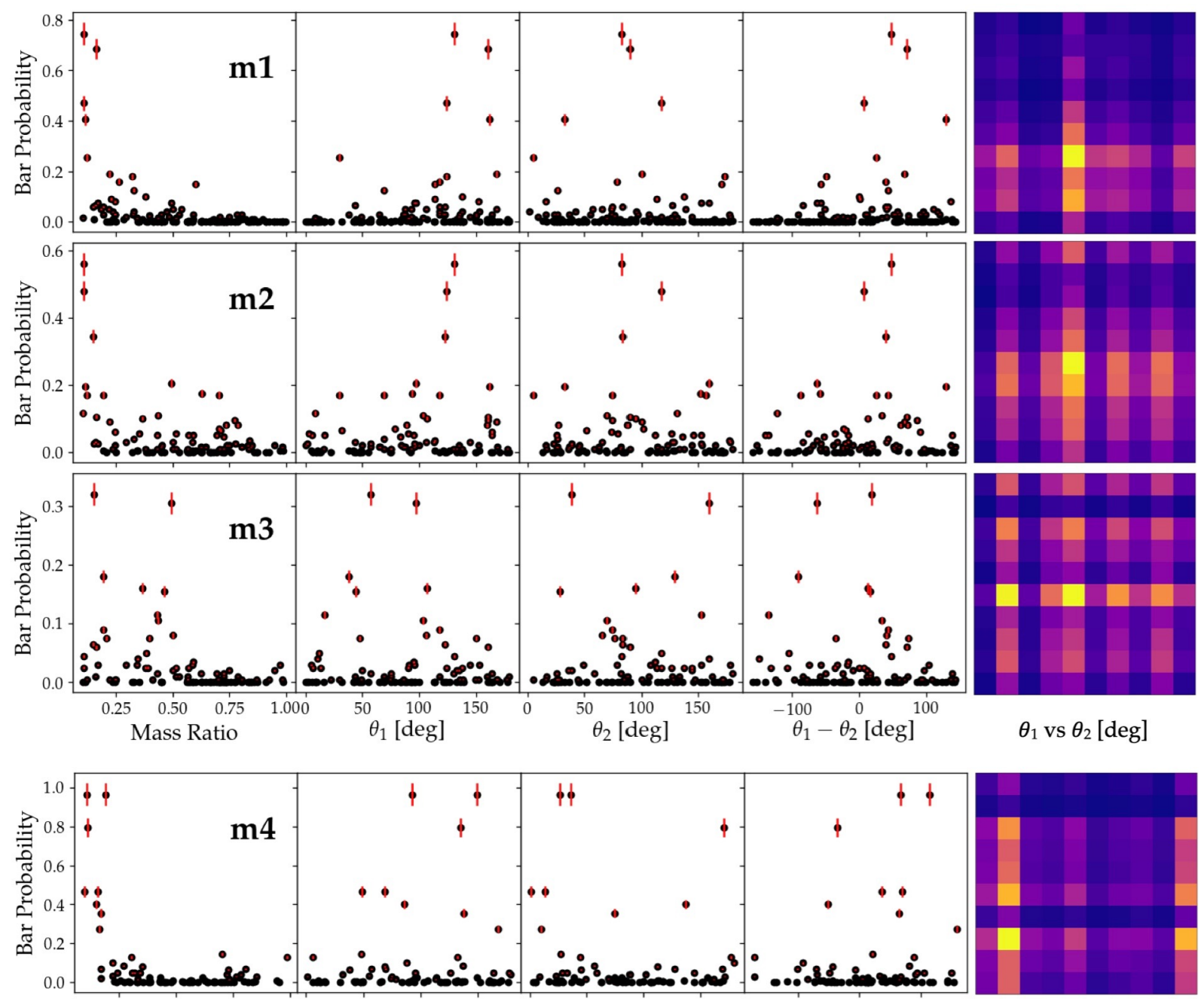

$\theta_{1}$ vs $\theta_{2}[\mathrm{deg}]$
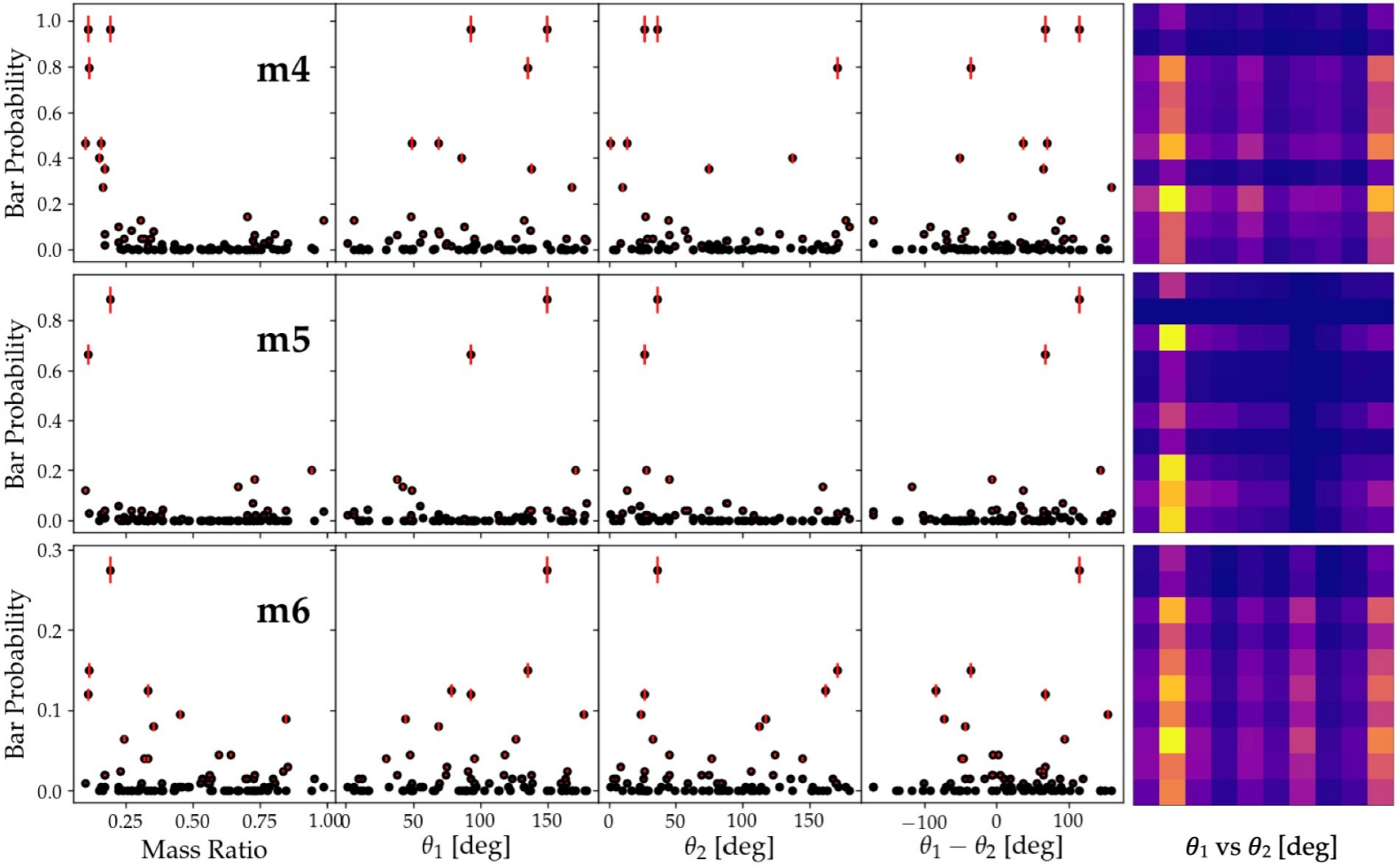

Fig. 4. Incidence of bar formation, expressed as the bar fraction $f_{\text {bar }}$ or bar probability, as a function of mass ratio $m_{2}$, the difference in spin angle $\theta_{1}, \theta_{2}$, and as a function of $\theta_{1}$ vs. $\theta_{2}$ where $\theta_{1}$ is on the vertical axis. Lighter shades in the normalised density map of $\theta_{1}$ vs. $\theta_{2}$ indicate a higher relative probability of bar detection. Error bars indicate one standard error $\sigma$.

points to qualitatively infer trends and pinpoint certain parameter ranges that are more conducive to bar formation than others.

In Fig. 4, we see both reciprocal relationships (such as the $\mathrm{m} 1$ model) and a relationship with two distinct peaks (the $\mathrm{m} 3$ model). This suggests that intermediate-mass mergers may be just as efficient as minor mergers at producing bars for certain galaxy models (in the case of $\mathrm{m} 3$, a galaxy model with a smaller stellar mass). For all models, major merging is far less conducive to bar formation compared to minor merging. Further simulations with a wider range of model parameters are required to definitively examine the mathematical nature of this relationship. 


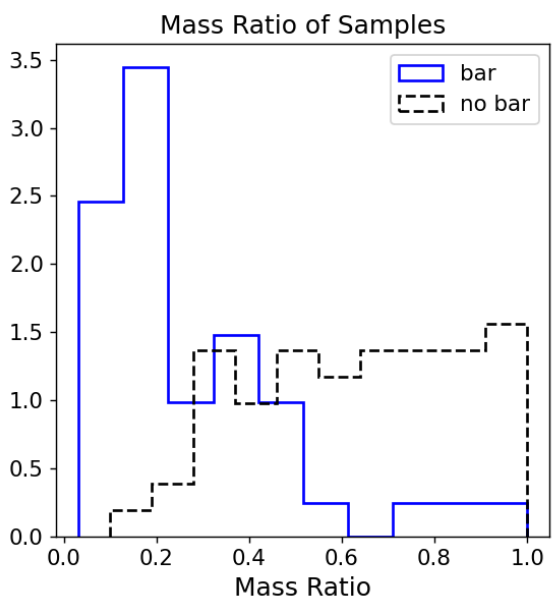

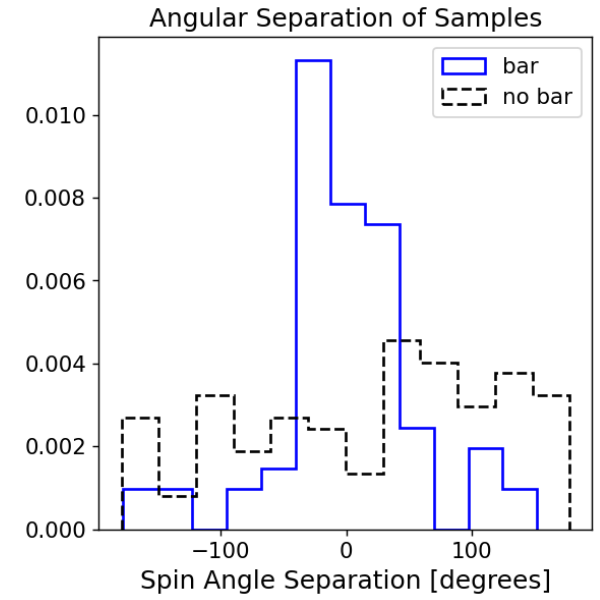

Fig. 5. Normalised histograms of all samples classified as either bar (blue) or no-bar (black, dotted) by our main $\mathrm{CNN}$, for different mass ratios (left) and spin angle separation (right).
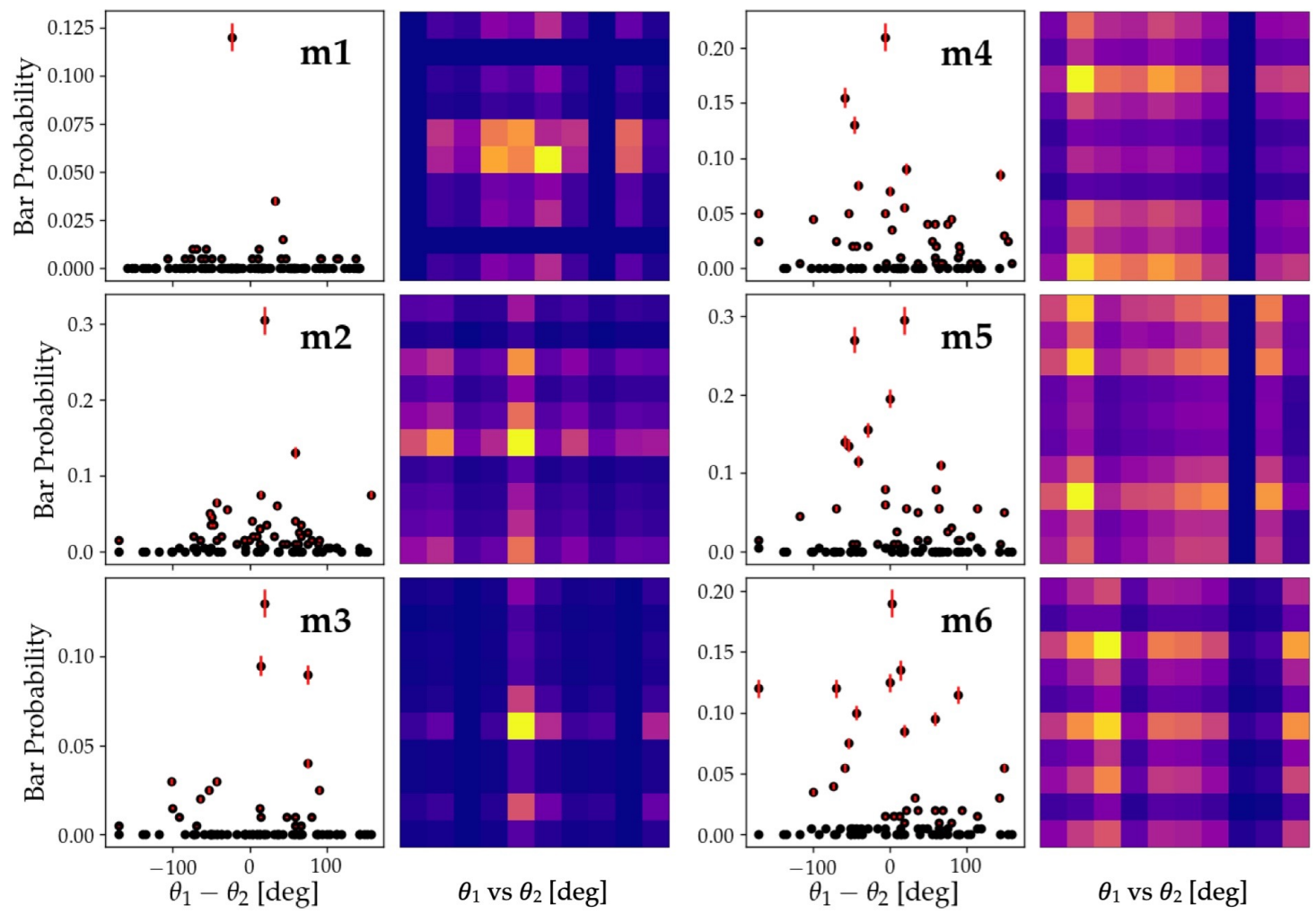

Fig. 6. Incidence of bar formation, expressed as the fraction of total images that were identified as bar for a given model, as a function of the difference in spin angles $\theta_{1}-\theta_{2}$ and as a function of spin angles $\theta_{1}$ vs. $\theta_{2}$ for major mergers at a fixed mass ratio of 1.0.

\subsection{Spin angles}

The $\mathrm{m} 1, \mathrm{~m} 2$ and $\mathrm{m} 3$ models appear to be more strongly constrained by $\theta_{1}$, while there is a dominant $\theta_{2}$ constraint for the $\mathrm{m} 4$, m5 and m6 models. Given the significant scatter in the overall spin angles, more simulations are needed to conclusively determine any specific constraints on either angle. Since the exchange of angular momentum is an important factor of bar formation, it is better to focus on the difference in the spin angles, $\theta_{1}-\theta_{2}$ (i.e. how closely aligned the discs are). We find that, for the $\mathrm{m} 1, \mathrm{~m} 2$ and $\mathrm{m} 3$ models, more bars were detected for more closely aligned $\theta_{1}$ and $\theta_{2}$ (i.e. $\left|\theta_{1}-\theta_{2}\right| \approx 0$ ) compared to mergers with wildly different spin angles. However, such a trend is not observed for the $\mathrm{m} 4, \mathrm{~m} 5$ and $\mathrm{m} 6$ models in the variable-mass case.
In order to analyse these spin angle constraints independently of mass ratios, we ran several models for all 6 of our galaxy merger models with a fixed mass ratio of 1.0 (Fig. 6), corresponding to major mergers. We previously established that fewer bars were detected in major merging; hence testing on major mergers yields a stronger constraint on the spin angles. Again, we find that models in which $\theta_{1}$ and $\theta_{2}$ are more closely aligned resulted in the detection of more bars. In each case, the parameter with the highest fraction of bar detection is located very close to $\theta_{1}-\theta_{2}=0$. This is also true for the $\mathrm{m} 4, \mathrm{~m} 5$ and m6 models. Importantly, these results were consistent across our various models despite their differences in bulge-to-disc ratios and dark matter contents. 

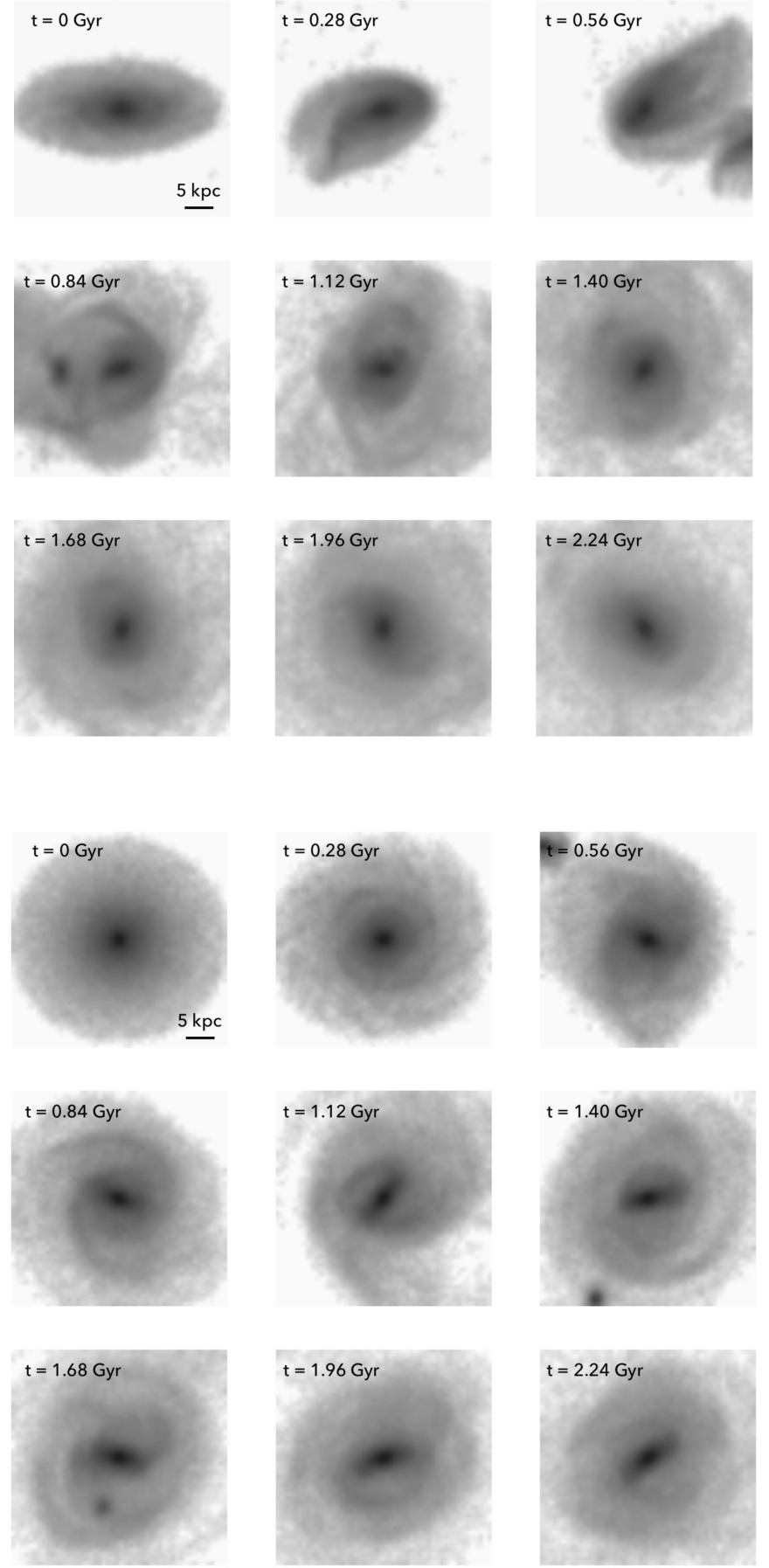

Fig. 7. Time evolution of the $2 \mathrm{D}$ normalised mass density distribution $\left(x-y\right.$ plane projection) for a major merger with mass ratio $m_{2}=1.0$ (top) and minor merger with mass ratio $m_{2}=0.1$ (bottom) for the small-bulge $\mathrm{m} 1$ model. Each frame measures $35 \mathrm{kpc}$ by $35 \mathrm{kpc}$ (these dimensions are used throughout) and each timestep is $240 \mathrm{Myr}$.

\section{Discussion}

To verify these constraints, we first identified several key cases that involved contrasting two simulation models. The most important of these test cases is to compare a major merger with a minor merger. We did this for the small-bulge $\mathrm{m} 1$ disc model (Figs. 7 and 8) as well the large-bulge $\mathrm{m} 4$ model with a bulgeto-disc ratio of 0.5 (Fig. 9). We decided to use the $\mathrm{m} 1$ model instead of the bulge-less m6 model, and the m4 model instead
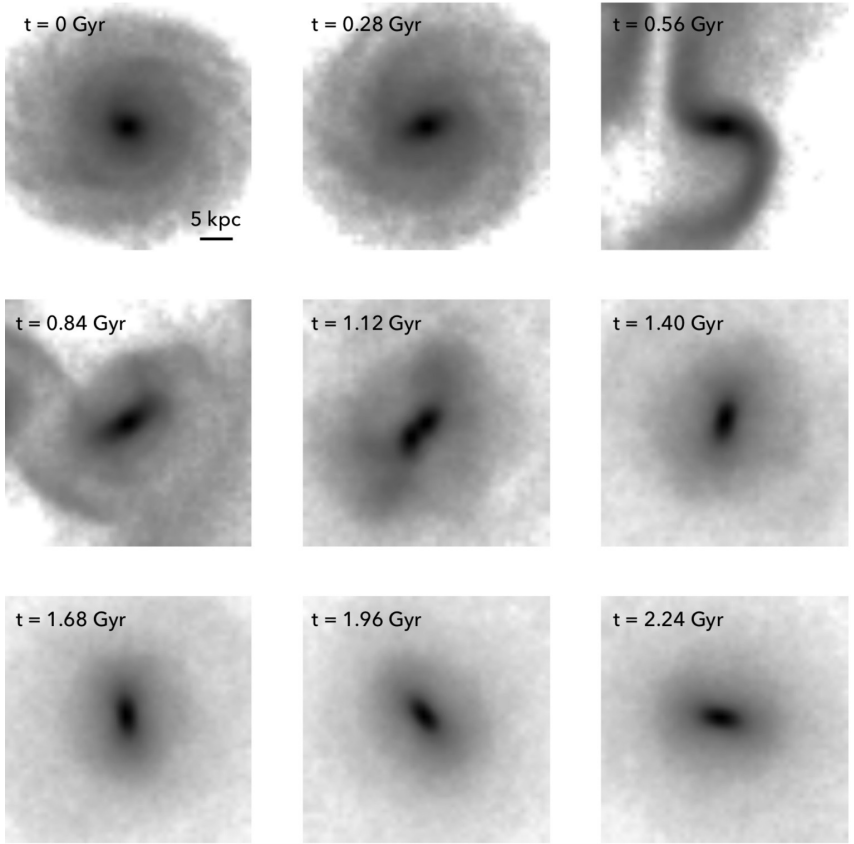

Fig. 8. Time evolution of the 2D normalised mass density distributions for our simulations of a major merger for $\theta_{1}, \theta_{2}=0$, demonstrating the formation of a bar. Frame dimensions are as in Fig. 7.

of the 1:1 bulge-to-disc m5 model, in order to keep in line with more realistic MW-type galaxies.

Since our models in Figs. 4 and 6 found that mergers with low angular separation were more conducive to bar formation, we also analysed a major merger for equal spin angles $\theta_{1}=\theta_{2}=0$. The latter of these cases is highly unrealistic given how statistically unlikely it is for two prograde galaxies to merge with the same orientation. We also compared a simulation of the $\mathrm{m} 4$ model in the isolated and minor merger case.

\subsection{The bar formation process}

It is clear from Fig. 7 that a stellar bar is readily formed in the minor merger case, while no bar is produced in the major merger. Importantly, the major and minor merger cases are distinguished by the timescale of the merger. In the major merger case, the galaxies merge destructively between $840 \mathrm{Myr}$ and $1.12 \mathrm{Gyr}$, while the minor merger completes between $1.68 \mathrm{Gyr}$ and $1.96 \mathrm{Gyr}$ (some $800 \mathrm{Myr}$ later). Our simulation results in Fig. 4 have shown that minor merging is more conducive to bar formation. This is important considering the well-studied effects of minor merging in the overall evolution of galaxies (Reichard et al. 2008). Simulations have also shown the vast majority of all mergers in the Universe to be minor mergers (Lotz et al. 2010). It is important, however, to keep in mind the low overall bar detections in Table 3; bar formation in galaxy merging is rare.

In most cases where a bar is detected in the aftermath of the merger event, the bar is first formed due to tidal interaction with the approaching galaxy, after which it then survives the merger process. In the case of major merging, the merger is destructive. Conversely, minor merging is constructive. This is sensible given the difference in gravitational effects. Smaller companions tend to slowly spiral into their parent (helping to induce a tidal bar), and by the time the merger is complete they are usually sufficiently stripped of mass so as to not warp or disturb the parent disc. Equal mass mergers are more destructive due to the quick 
head-on collision (such as what we see in Fig. 7) that severely warps the stellar disc.

This latter notion of whether a bar either survives or is destroyed is the key motivation behind finding constraints for our simulation parameters. These constraints will help guide further investigation into the exact mechanisms with which bars can survive a galaxy merger unscathed. It is important to note that there are two mechanisms at play here; the initial pre-merger tidal interactions, and the actual merger itself. Previous studies such as Peirani et al. (2009) and Di Matteo et al. (2010) have shown that bars can form due to the tidal interactions before a merger, however they did not consider what happens to the bar during the merger itself. To better illustrate this dual-nature, Fig. 8 shows a major merger with $\theta_{1}=\theta_{2}=0$ degrees.

First and foremost, Fig. 8 shows bar formation in the major merger case. So although the results of Fig. 4 show that minor merging is much more promising, we have demonstrated that bar formation is still possible for major mergers. The spin angles (i.e the orientation of the galaxies) are also key to whether or not a bar is produced. In Fig. 8, we see that a bar is induced at around $0.56 \mathrm{Gyr}$. The actual merger takes place between 0.84 and 1.12 Gyr. However, the snapshot at 1.12 Gyr shows the bar has buckled into two loosely connected bulges (likely due to the force of the merger), but by $1.40 \mathrm{Gyr}$ the stellar bar has reformed and continues to exist for the rest of the simulation timescale. This is strong evidence for the role of spin angles in the formation and survival of stellar bars in merging. So while it is possible for bars to be induced prior to the merger due to tidal interactions, regardless of orientation (as tidal interactions are gravitational in nature), orientation is key to whether a bar can be reformed in the aftermath of a galaxy merger. It is this latter mechanism that we wish to highlight in this current study.

Angular momentum is a key factor in galaxy merging (Athanassoula 2005; Pedrosa \& Tissera 2015). The results of Figs. 6 and 8 show that major mergers with more closely aligned orientations (i.e. $\theta_{1}-\theta_{2} \mid=0$ ) have higher bar detection rates. This is likely due to a more favourable transfer of angular momentum that, as Fig. 8 shows, is sufficient to regenerate the stellar bar.

Thus we have shown that there are two distinct phases that govern the overall bar formation process in galaxy merging; (1) pre-merger tidal interactions, and (2) reformation of the bar during and/or after the merger. Our results have shown that the minor merging of two galaxies with near-identical spin angles is most conducive to bar formation in the aftermath of a merger. This highlights the importance of angular momentum transfers, and also the role of gravitational forces given the sharp decline in bar detections as the mass ratio increases. We expect that the second phase is more dominant in major merging due to the destructive nature of the merger whereby strong gravitational forces greatly perturb the stellar disc.

\subsection{Effect of stellar bulges}

The bar fractions in Table 3 are lower for models with smaller bulges. This is somewhat counter-intuitive given that bulges tend to suppress bar formation in isolation. This suggests that a bulges may play a more important role in galaxy merging. To visualise whether stellar bulges have a meaningful impact on bar formation, we ran a test simulation with the $\mathrm{m} 4$ model (bulgeto- disc ratio of 0.5 ) for both a major and minor merger (see Fig. 9). As anticipated (based on the results of Fig. 4 and the bar fractions in Table 3), major merging is destructive while minor merging is constructive. A key physical observation is that the addition of a sizeable stellar bulge has increased the time taken for a bar to be induced. In the minor merging case, a bar is only induced after around 1.68 to $1.96 \mathrm{Gyr}$ (considerably more time than in Fig. 7). This supports the well-known dynamical role that stellar bulges play in stabilising disc instabilities (Kataria \& Das 2017) and hence inhibiting bar growth (Sellwood \& Wilkinson 1993).

\subsection{Comparison to the isolated case}

To better understand the role of minor merging in bar formation, we ran a simulation of the $\mathrm{m} 4$ galaxy model in both the isolated case and for a minor merger with mass ratio $m_{2}=0.1$ (see Fig. 10). The idea is that the bulge should suppress bar formation, yet in the minor merging case of Fig. 10, we see that bar is induced as early as $1.12 \mathrm{Gyr}$, while there is only evidence of a weak bar in the isolated case from roughly 1.96 Gyr onwards. The bar formed in the minor merger is more well defined and is also accompanied by spiral arms.

That minor merging can induce bars in bulge-dominant discs is particularly important since it thought that bulges can stabilise disc instabilities (Binney \& Tremaine 2008), particularly in isolation. Kataria \& Das (2017) investigated upper limits on bulge-to-disc ratios for bar formation in isolation, finding that massive bulges stabilise the disc by cutting off angular momentum exchange between the disc and halo.

Lotz et al. (2010) investigated the role of mass ratios in galaxy morphology with cosmological simulations and note that the vast majority of galaxy mergers will be minor mergers rather than equal-mass major merging. In terms of morphological evolution, minor merging can lead to morphological distortion (Reichard et al. 2008) that ultimately affects galaxy properties (Darg et al. 2010). Minor merging is a crucial process in the formation and evolution of galaxies. We have found that mass ratio indeed plays a key role in bar formation, confirming the importance of mass ratio when studying the processes that govern galaxy merger.

\subsection{Pattern speeds}

One important factor to ensure the accuracy of the classifications is to determine whether the bars as seen in Figs. 7, 9 and 10 are actually real bars, or instead just elongated bulges. One such method is to analyse the pattern speed $\Omega_{b}$, which is the rate (or frequency) with which the bar rotates. The pattern speed is a very important parameter when it comes to the dynamics of barred galaxies (Sellwood \& Wilkinson 1993; Sellwood \& Sparke 1988; Athanassoula 2005). This is since rotation is a key physical feature of bars (Sellwood \& Wilkinson 1993) that distinguishes them from elongated bulges or other irregular morphological features. There are many well established methods to analyse pattern speeds (Gerssen et al. 2003; Aguerri et al. 2015; Wu et al. 2018b) including the classic kinematic-based approach of Tremaine \& Weinberg (1984; also known as the TW method). The TW method is heavily reliant on additional data such as lineof-sight velocity maps and is more suited to observational data (see Aguerri et al. 2015).

\section{Classifying bar formation mechanisms}

In this section, we discuss our secondary aim of classifying an image of a barred galaxy according to the mechanism with which it was formed. As this is a separate $\mathrm{CNN}$ from our main $\mathrm{CNN}$, we refer to it as the "bar-type" CNN. Figure 11 illustrates the 
M. K. Cavanagh and K. Bekki: Bar formation with deep learning
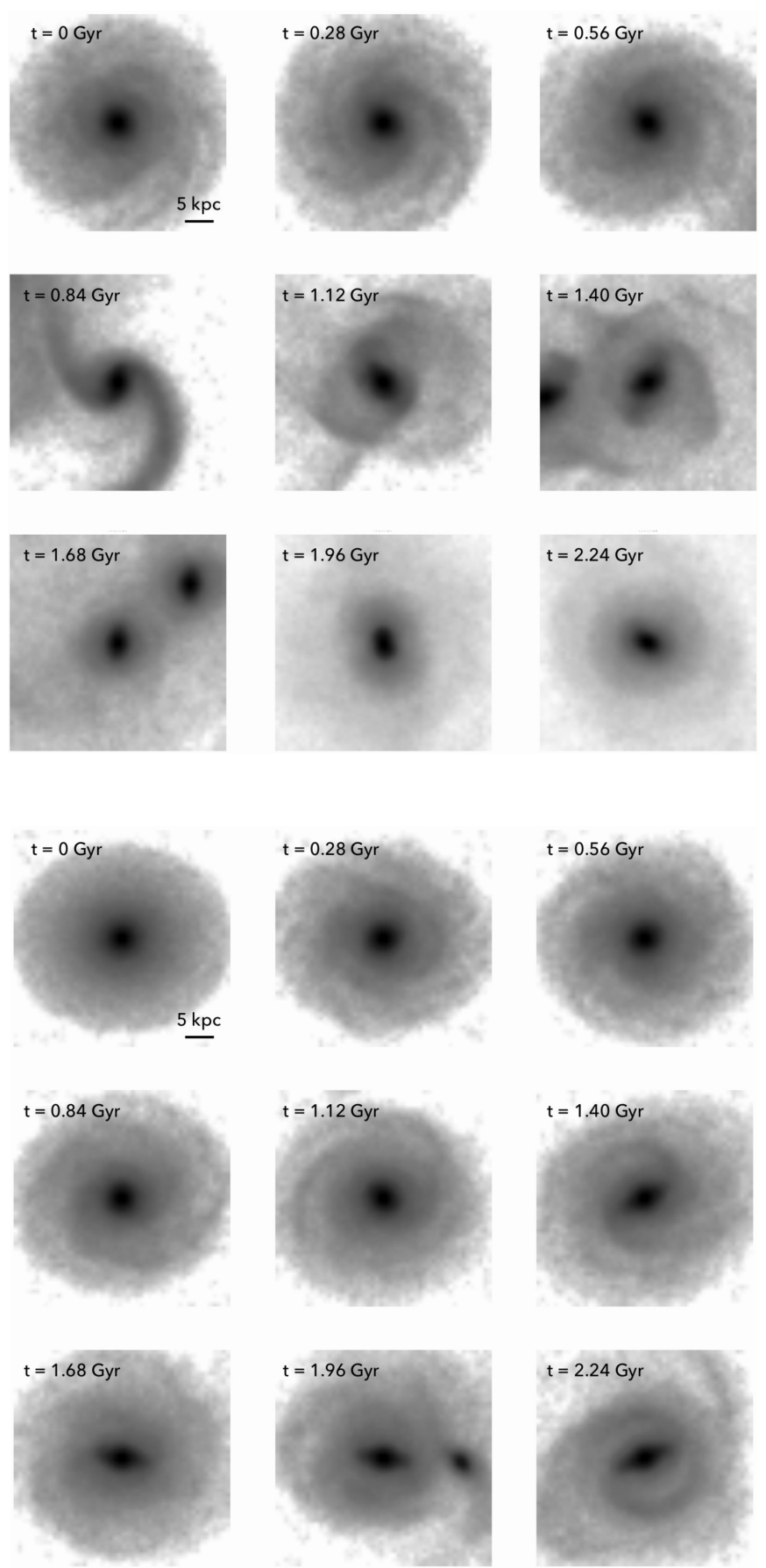

Fig. 9. Time evolution of our simulations of a major merger with mass ratio $m_{2}=1.0$ (top) and a minor merger with mass ratio $m_{1}=0.1$ for a model with a bulge-to-disc ratio of 0.5 (bottom).

rationale for this aim. It shows images of galaxies from our simulations in the isolated, tidal and minor merger cases, as well as an image with no bar. Just as the human eye can discern between these four cases, we wish to determine whether a CNN can do the same. For this step, we use the same CNN architecture used for our main analysis, albeit instead of 2 nodes in the output layer there are now 4 (corresponding to the 4 categories). This new network was trained with a much smaller training set of 4800 images obtained from running the $\mathrm{m} 1, \mathrm{~m} 2$ and $\mathrm{m} 3$ models for the isolated, tidal and merger case (with images that showed no bar formation added to the "no- bar" category). This smaller set was visually classified up to an accuracy of $100 \%$ with both
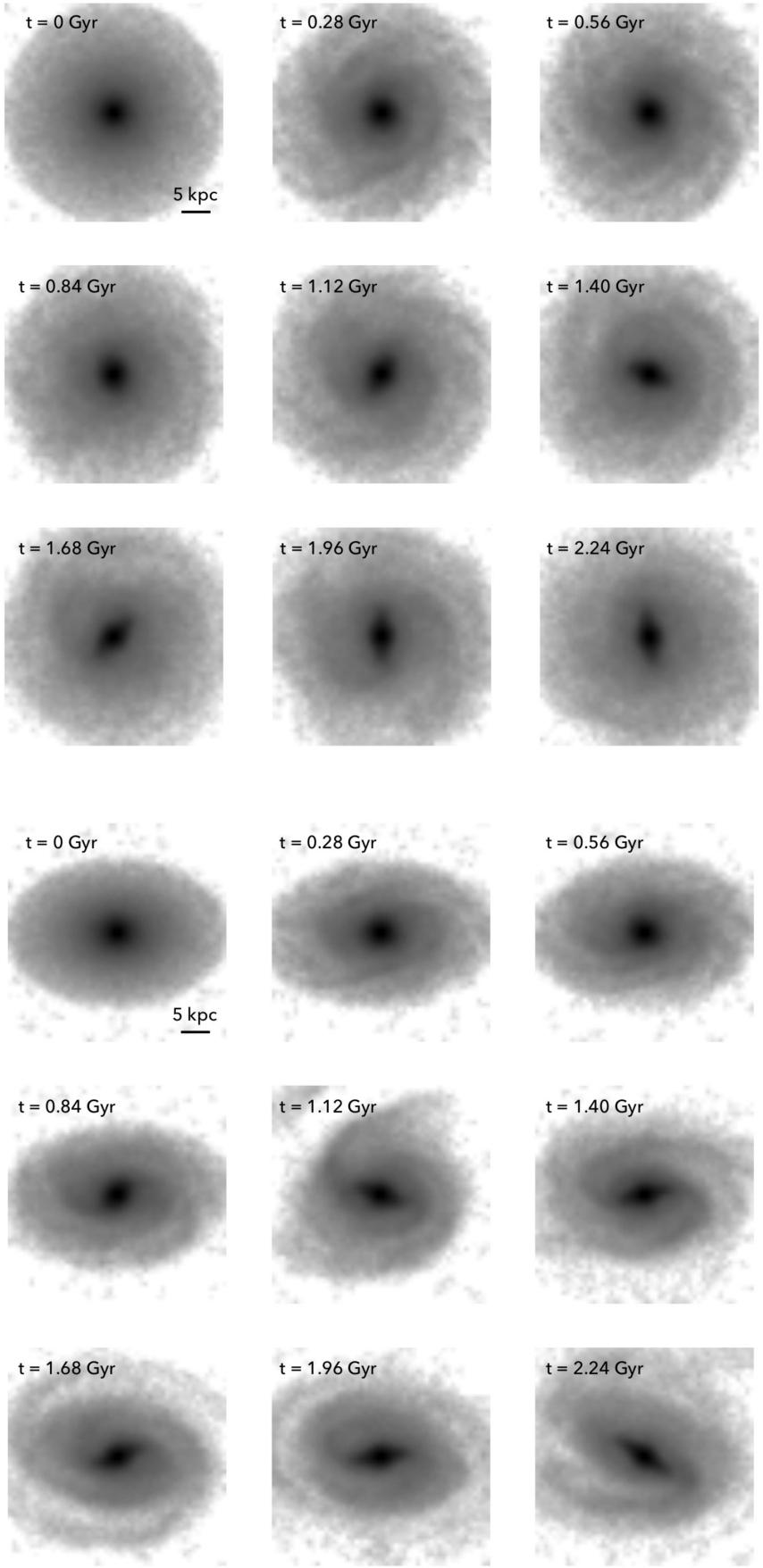

Fig. 10. Time evolution of our simulations of a galaxy with a bulgeto-disc ratio of 0.5 in the isolated case (top) and merger case (bottom).

observers agreeing on all classifications, however it is likely a larger set would be subject to more uncertainty. We randomly partitioned this training set such that $80 \%$ of the samples were used the train the network, with the remaining $20 \%$ used for validation purposes. The network was trained up to maximum total accuracy over 300 epochs with the same internal architecture as the main CNN albeit it with four output nodes instead of two.

One way to determine how accurate the CNN is at distinguishing between multiple categories is by looking at the confusion matrix (or error matrix). The confusion matrix lists the actual categories and predicted categories of a set of samples. Assuming an 100\% accurate network, the confusion matrix should be a diagonal matrix. 


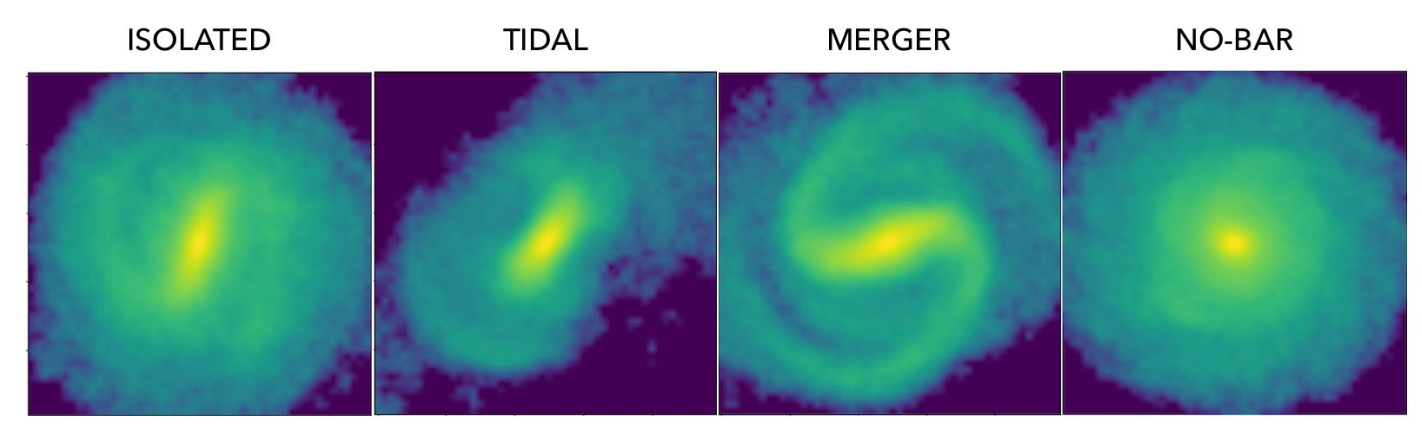

Fig. 11. Images in each of the four different morphological categories considered for our secondary, bar-type CNN.

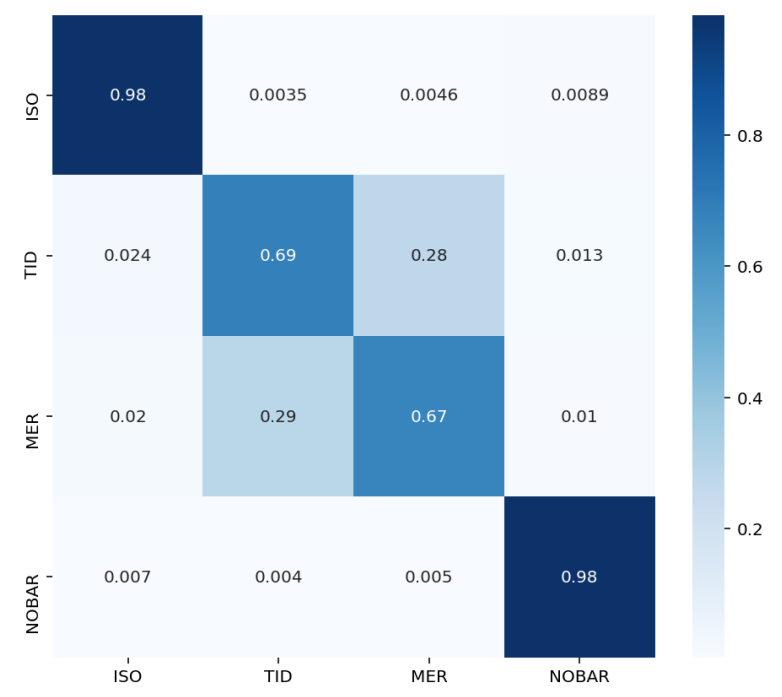

Fig. 12. Confusion matrix of classification accuracies for our CNN Here the columns represent the actual category and the rows represent the predicted category.

As can be seen from the confusion matrix of our bar-type $\mathrm{CNN}$ in Fig. 12, there were mixed results. While testing accuracies for the isolated bar and no-bar case were consistently above $98 \%$, the accuracy dropped off to $68 \%$ and $67 \%$ for the tidal bars and merger bars respectively (Fig. 12). This is likely due to their morphological similarities (see Fig. 11). Since isolated bar formation is generally not accompanied by morphological features such as tidal tails or otherwise distorted discs, it is much easier to distinguish between both isolated bars and tidal bars, and isolated bars and merger bars. Comparing tidal bars and merger bars is not as straightforward since both share significant morphological distortion. This can be seen in the values in the confusion matrix (Fig. 12) where the rates of misclassification are well below $1 \%$ for the other 3 cases, and also why tidal bars were classified as merger (and vice versa) with accuracies of $29 \%$ (and 28\%).

An alternative method to discriminate between tidal and merger bars may be to compare the kinematics as given by 2D maps of the line- of-sight velocity dispersion. Neural networks trained on kinematic data may more accurately distinguish between tidal and merger bars. This is since the velocity dispersion is much more uniform in tidally barred galaxies, since the structure of the galaxy remains for the most part intact, while for merger galaxies there is significant distortion and irregularities in the velocity dispersion due to the impact of the collision. So although the normalised mass distribution may show similar morphological features in tidal and merger bars, the differences may only become apparent when considering the kinematic data. This is a promising consideration for future studies.

\subsection{Use of neural networks}

As our analysis is heavily reliant on the use of neural networks, it was important to ensure that the networks were accurate. In general, the performance and accuracy of neural networks is largely determined by both the quantity and quality of the training set. Memory limitations on the Pleiades cluster necessarily impose an upper limit on the amount of data that we can train with, while limits on the visual classification process introduce an inherent uncertainty in the training set's accuracy.

We found evidence of misclassification occurring with some major mergers where, although the final image was classified as a bar, in reality the image was of a non-rotating, prolate bulge (an example of this situation can be seen in the major merger result of Fig. 7). One of the key limitations of this deep learning approach is that the classification of galaxies is based on a single, static image; hence ignoring the need for bars to rotate.

An important issue arose with images showing near edgeon galaxies. Here the corresponding probability approached 50-50 bar and no-bar; a random classification. A hasty solution to this may well be to restrict the outputs to solely face-on orientations with rotations only, however to do so would severely limit the usefulness of the CNN and its ability to mirror realworld imagery. Instead, a combination of a higher resolution for the input image, coupled with a much larger training set, would likely result in improved performance at high inclinations. The case of perfectly edge-on galaxies would likely still remain close to random as, especially for the case of isolated bars where there is little morphological distortion, there is simply not enough information in the image for the various convolution layers to work with. Despite this, it may be feasible to classify bars according to their peanut or X-shape (Raha et al. 1991; Pérez et al. 2017) when viewed edge-on. We also encountered a case of incorrect classification in the case of an image of a spiral arm with no stellar bar, Although this was only observed in one case out of the hundreds of visually verified cases, it is still an issue worth noting. It may be possible that the $\mathrm{CNN}$ confused the presence of spiral arms with that of a bar. The solution to this would be to explicitly include spiral-only samples labelled as "no-bar" in the training set.

It is also important to consider the timescale used in the $N$-body simulations. Although after $2.24 \mathrm{Gyr}$ we see clear morphological distinctions between the different types of bars, we have not trained the CNN on any images beyond this timescale. Another consequence of using the final time-step image as input to the $\mathrm{CNN}$ is that bars that survived the merger could have been 
destroyed in post-merger interactions before the end of the simulation. Although we have not seen direct visual evidence for this, we cannot rule out bar destruction several hundreds of Myr after the merger completes. Thus the obtained $f_{\text {bar }}$ values in Table 3 may have been higher were the CNN to have focused on the timestep immediately following the merger rather than the final timestep after around 2.24 Gyr. In practice, this is near impossible to implement for it either requires an accurate prediction of the merger timescale in advance, or the manual inspection of each model (defeating the purpose of using neural networks). Were a CNN to be trained using images from all timesteps, it may be possible to examine the rates of bar formation as a function of time, however the timestep must be small enough to capture the immediate aftermath of the galaxy merger.

Despite these issues, CNNs are an incredibly powerful tool that have the potential to revolutionise the speed with which large-scale imaging surveys can be processed and analysed. There are many benefits of deep learning-based approaches to observational studies, particularly since deep learning is a general paradigm whereby the neural networks can be easily tailored to the task at hand. Furthermore, a CNN that can classify barred galaxies according to the mechanism with which they formed would be a boon for studies into the environmental dependence of bars. Automated galaxy classification based on machine learning is a promising method with which to quickly parse large-scale data and accelerate studies into galaxy formation and evolution. However, as with all applications of machine learning, there is the important caveat that the overall performance of the neural network is dependent on the accuracy, quality and relative size of the data on which it is trained (Haykin 2009). As modern digital surveys yield increasingly larger datasets, the suitability and accuracy of neural networks and other deep learning methods will only increase.

\subsection{Future considerations}

We established our results using $N$-body simulations rather than using hydrodynamic simulations that include gas. A key reason for this was to simplify the overall simulation, lowering the time it takes to evolve a single galaxy. This is important since, in order to obtain the trends in Figs. 4 and 6, it was necessary to run thousands of models, with each $N$-body simulation taking a considerable amount of time to complete depending on stellar mass and bulge mass. Adding gas would greatly increase the time taken to simulate each sample, making it impossible to carry out this work. It is known that gas plays an important role in the overall evolution of stellar bars (Berentzen et al. 1998, 2007; Bournaud \& Combes 2002; Bournaud et al. 2005), particularly in gas-rich isolated discs where the gas can act as a stabilising force, reduce the rate bar formation, and can result in weaker bar (Athanassoula et al. 2013). However, since the main focus of this work is on bar formation in galaxy mergers where much gas stripping occurs, we have decided to use $N$-body simulations and omit tracking gas particles entirely. Other future considerations include utilising and/or training a new $\mathrm{CNN}$ to be used with observational surveys in order to determine the prevalence of different types of bars throughout the Universe. Such studies could also determine how the bar fraction changes due to environment or redshift.

\section{Conclusions}

Through our $N$-body simulations, we have investigated the formation of bars via three different formation mechanisms; the iso- lated (spontaneous) model, the tidal interaction model, and the galaxy merger model. We have designed and trained a convolutional neural network to identify the presence of bars based on two-dimensional normalised density maps. We have investigated how the fraction of bars detected by our $\mathrm{CNN}, f_{\mathrm{bar}}$, changes as we vary the mass ratio $m_{2}$ and spin angles $\theta_{1}, \theta_{2}$. We have been able to determine the parameter ranges most conducive to bar formation for multiple models that differ in both dark matter content and bulge-to-disc ratios. Through extending our CNN to include multiple labels, we have shown that it is feasible for a CNN to classify a bar according to the mechanism with which it was formed. There are several important conclusions that we reached based on our work:

(1) We have found that minor mergers (i.e. those with $m_{2} \approx 0.1$ ) with similar orientations $\left(\theta_{1} \approx \theta_{2}\right)$ are most conducive to bar formation.

(2) Through visualising the bar formation process, we have found that there are two distinct phases. The first phase involves the formation of a tidally induced bar due to premerger interactions as the two galaxies spiral into each other. This is more likely to occur in the minor merger case as the merger timescale is longer. The second phase involves whether or not the bar survives the merger or, in the case of a destructive major merger, whether the bar can regenerate. Minor merging typically preserves the bar, whilst major merging destroys the bar. By showing that is is possible for a bar to regenerate in the case of equal spin angles $\theta_{1}=\theta_{2}=0$ degrees (Fig. 8), we infer that the transfer of angular momentum is key to the regeneration of the bar.

(3) It is possible for strong bars to form in minor merging in cases where bars are otherwise suppressed in isolation due to the presence of a large stellar bulge (Fig. 10). This suggests that bulges may help to facilitate bar formation in galaxy merging.

(4) We found that some galaxy models in Table 2 that failed to produce strong bars in the isolated case produced strong bars in galaxy merging. Thus galaxy merging can enhance bar formation. This has several implications, for instance bars at high redshift $z$ can be triggered by early minor merging. This is also important for SB0 galaxies where it is thought that merging is key to their formation.

(5) We have shown that is feasible for a CNN to classify multiple bar formation mechanisms, however this was achieved using a much smaller training set than that employed for our main analysis. We found a higher accuracy when classifying isolated bars, albeit tidal and merger bars were more difficult to distinguish.

Through techniques from deep learning, we have successfully shown that bars can be produced in simulated galaxy mergers. Despite their limitations, CNNs are a versatile tool that can be used to rapidly analyse and classify the output of galaxy simulations.

Acknowledgements. We are grateful to the referee for their comments and constructive feedback that helped to improve this paper.

\section{References}

Abraham, S., Aniyan, A. K., Kembhavi, A. K., Philip, N. S., \& Vaghmere, K. 2018, MNRAS, 477, 894

Aguerri, J. A. L., Beckman, J. E., \& Prieto, M. 1998, AJ, 116, 5

Aguerri, J., Méndez-Abreu, J., \& Corsini, E. 2009, A\&A, 495, 491

Aguerri, J. A. L., Méndez-Abreu, J., Falcón-Barroso, J., et al. 2015, A\&A, 576, A102

Athanassoula, E. 1999, Astrophysical Discs - an EC Summer School, eds. J. A. Sellwood, \& J. Goodman, 351 
Athanassoula, E. 2003, MNRAS, 341, 1179

Athanassoula, E. 2005, Celestial Mech. Dyn. Astr., 91, 9

Athanassoula, E., Bienayme, O., Martinet, L., \& Pfenniger, D. 1983, A\&A, 127, 349

Athanassoula, E., Machado, R. E. G., \& Rodionov, S. A. 2013, MNRAS, 429, 1949

Barberà, C., Athanassoula, E., \& García-Gómez, C. 2004, A\&A, 415, 849

Barnes, J. 1996, New Light on Galaxy Evolution, eds. J. Barnes, \& R. L. Davies, IAU Symp., 171, 191

Barnes, J. E., \& Hernquist, L. 1992, ARA\&A, 30, 705

Bekki, K. 2013, MNRAS, 432, 2298

Bekki, K., Diaz, J., \& Stanley, N. 2019, A\&C, 2800286

Berentzen, I., Heller, C. H., Shlosman, I., \& Fricke, K. J. 1998, MNRAS, 300, 49

Berentzen, I., Athanassoula, E., Heller, C. H., \& Fricke, K. J. 2004, MNRAS, 347,220

Berentzen, I., Shlosman, I., Martinez-Valpuesta, I., \& Heller, C. H. 2007, ApJ, 666,189

Binney, J., \& Tremaine, S. 2008, Galactic Dynamics, 2nd edn. (Princeton University Press)

Bournaud, F., \& Combes, F. 2002, A\&A, 392, 83

Bournaud, F., Combes, F., \& Semelin, B. 2005, MNRASL, 364, L18

Bridge, C. R., Appleton, P. N., Conselice, C. J., et al. 2007, ApJ, 659, 931

Calleja, J. D. L., \& Fuentes, O. 2004, MNRAS, 349, 87

Cattaneo, A., Mamon, G. A., Warnick, K., \& Knebe, A. 2011, A\&A, 533, A5

Chollet, F. 2015, Keras, https: //keras. io

Conselice, C. J. 2014, ARA\&A, 52, 291

Consolandi, G. 2016, A\&A, 595, A67

Contopoulos, G., \& Grosbøl, P. 1989, A\&ARv, 1, 261

Dalcanton, J., Yoachim, P., \& Bernstein, R. 2004, ApJ, 608, 189

Darg, D. W., Kaviraj, S., Lintott, C. J., et al. 2010, MNRAS, 401, 1552

Di Matteo, P., Qu, Y., Lehnert, M. D., van Driel, W., \& Jog, C. J. 2010, EAS Pub. Ser., 45, 389

Diaz, J. D., Bekki, K., Forbes, D. A., et al. 2019, MNRAS, 486, 4845

Dieleman, S., Willett, K. W., \& Dambre, J. 2015, MNRAS, 450, 1441

Elmegreen, D. M., Bellin, A. D., \& Elmegreen, B. G. 1990, ApJ, 364, 415

Eskridge, P. B., \& Frogel, J. A. 1999, Ap\&SS, 269, 427

Eskridge, P. B., Frogel, J. A., Pogge, R. W., et al. 2000, AJ, 119, 536

Fanali, R., Dotti, M., Fiacconi, D., \& Haardt, F. 2015, MNRAS, 454, 3641

Friedli, D., \& Benz, W. 1993, A\&A, 268, 65

Gadotti, D. 2011, MNRAS, 415, 3308

Garcia-Gómez, C., \& Athanassoula, E. 1991, A\&AS, 89, 159

Garcia-Gómez, C., Athanassoula, E., Barberà, C., \& Bosma, A. 2017, A\&A, 601, A132

Gerssen, J., Kuijken, K., \& Merrifield, M. R. 2003, MNRAS, 345, 261

Goldreich, P., \& Lynden-Bell, D. 1965, MNRAS, 130, 125

Graff, P., Feroz, F., Hobson, M. P., \& Lasenby, A. 2014, MNRAS, 441, 1741

Haykin, S. O. 2009, Neural Networks and Learning Machines, 3rd edn. (Pearson)

Hernquist, L., \& Mihos, J. C. 1995, ApJ, 448, 41

Hohl, F. 1971, ApJ, 168, 343

Huertas-Company, M., Rodriguez-Gomez, V., Nelson, D., et al. 2019, MNRAS, 489,1859
Julian, W. H., \& Toomre, A. 1966, ApJ, 146, 810

Kataria, S. K., \& Das, M. 2017, MNRAS, 475, 1653

Kaviraj, S., Peirani, S., Khochfar, S., Silk, J., \& Kay, S. 2009, MNRAS, 394, 1713

Kaviraj, S., Devriendt, J., Dubois, Y., et al. 2015, MNRAS, 452, 2845

Kormendy, J., \& Kennicutt, R. C., Jr 2004, AR\&A, 42, 603

LeCun, Y., Bottou, L., Bengio, Y., \& Haffner, P. 1998, Proc. IEEE, 86, 2278

LeCun, Y., Bengio, Y., \& Hinton, G. 2015, Nature, 512, 436

Lin, L., Koo, D. C., Weiner, B. J., et al. 2007, ApJ, 660, L51

Little, B., \& Carlberg, R. G. 1991, MNRAS, 250, 161

Lotz, J. M., Jonsson, P., Cox, T. J., \& Primack, J. R. 2010, MNRAS, 404, 575

Lukic, V., Brüggen, M., Mingo, B., et al. 2019, MNRAS, 487, 1729

Lynden-Bell, D. 1979, MNRAS, 187, 101

Lynden-Bell, D., \& Kalnajs, A. J. 1972, MNRAS, 157, 1

Mayer, L., Mastropietro, C., Wadsley, J., Stadel, J., \& Moore, B. 2006, MNRAS, 369, 1021

Miwa, T., \& Noguchi, M. 1998, ApJ, 499, 1

Moetazedian, R., Polyachenko, E. V., Berczik, P., \& Just, A. 2017, A\&A, 604, A75

Naim, A., Lahav, O., Sodre, L., \& Storrie-Lombardi, M. C. 1995, MNRAS, 275, 567

Navarro, J. F., Frenk, C. S., \& White, S. D. M. 1996, ApJ, 462, 563

Negroponte, J., \& White, S. D. M. 1983, MNRAS, 205, 1009

Neto, A. F., Gao, L., Bett, P., et al. 2007, MNRAS, 381, 1450

Noguchi, M. 1987, MNRAS, 228, 635

Pearson, W. J., Wang, L., Alpaslan, M., et al. 2019, A\&A, 631, A51

Pedrosa, S. E., \& Tissera, P. B. 2015, A\&A, 584, A43

Peirani, S., Hammer, F., Flores, H., Yang, Y., \& Athanassoula, E. 2009, A\&A, 496, 51

Pérez, I., Martínez-Valpuesta, I., \& Ruiz-Lara, T. 2017, MNRAS, 470, L122

Pfenniger, D. 1984, A\&A, 134, 373

Pfenniger, D., \& Friedli, D. 1991, A\&A, 252, 75

Prieto, M., Aguerri, J., Varela, A., \& noz Tuñón, C.M. 2001, A\&A, 367, 405

Raha, N., Sellwood, J. A., James, R. A., \& Kahn, F. D. 1991, Nature, 352, 411

Rautiainen, P., Salo, H., \& Laurikainen, E. 2002, MNRAS, 337, 1233

Regan, M. W., \& Teuben, P. 2003, ApJ, 582, 723

Reichard, T. A., Heckman, T. M., Rudnick, G., Brinchmann, J., \& Kauffmann, G. 2008, ApJ, 677, 186

Sellwood, J. A. 2014, Rev. Mod. Phys., 86, 1

Sellwood, J. A., \& Sparke, L. S. 1988, MNRAS, 231, 25P

Sellwood, J. A., \& Wilkinson, A. 1993, Rep. Prog. Phys, 56, 173

Sparke, L. S., \& Sellwood, J. A. 1987, MNRAS, 225, 653

Springel, V., \& Hernquist, L. 2005, ApJ, 622, L9

Toomre, A. 1964, ApJ, 139, 1217

Toomre, A., \& Toomre, J. 1972, ApJ, 178, 623

Tremaine, S., \& Weinberg, M. D. 1984, ApJ, 282, L5

Vasconcellos, E. C., de Carvalho, R. R., Gal, R. R., et al. 2011, AJ, 141, 189

Vera, M., Alonso, S., \& Coldwell, G. 2016, A\&A, 595, A63

Wu, C., Wong, O. I., Rudnick, L., et al. 2018a, MNRAS, 482, 1211

Wu, Y.-T., Pfenniger, D., \& Taam, R. E. 2018b, ApJ, 860, 152

Zeiler, M. D. 2012, ArXiv e-prints [arXiv:astro-ph/1212 . 5701] 